\title{
La actitud fenomenológica de la epojé en un estudio sobre participación política de mujeres jóvenes
}

\author{
Pilar Folgueiras-Bertomeu \\ Universitat de Barcelona. España. https://orcid.org/0000-0002-1343-0312 \\ Valeria de-Ormaechea-Otalora \\ Universitat de Barcelona. España. https://orcid.org/0000-0002-5742-4408 \\ Ariadna Salvà-Aige \\ Universitat de Barcelona. España. https://orcid.org/0000-0001-7323-9344 \\ Míriam Comet-Donoso \\ Universitat de Barcelona. España. https://orcid.org/0000-0003-2738-6217 \\ Correo para la correspondencia: miriamcomet@ub.edu
}

Artículo metodológico. Recibido: 03/07/2021. Aceptado: 07/10/2021. Publicación avanzada: 24/11/2021. Publicación: 03/01/2022.

\begin{abstract}
Resumen
INTRODUCCIÓN. El enfoque fenomenológico comporta una exploración constante. Para ello resulta imprescindible la revisión del equipo de investigación acerca de los preconceptos que están funcionando en relación con el tema de estudio. La epojé tiene un papel fundamental para comprender la participación política siendo mujer y joven. El proceso de revisión constante de las ideas y prejuicios es la parte central en la investigación fenomenológica.
\end{abstract}

MÉTODO. Se exponen las cuatro fases del proceso de investigación para detenernos en la primera parte de la segunda fase; en concreto, en la experiencia de participación política del equipo de investigación. Las reflexiones sobre las experiencias de las integrantes del equipo se realizan a partir de relatos y preguntas abiertas y reuniones sobre participación política.

RESULTADOS y DISCUSIÓN. Las ideas y los prejuicios del equipo de investigación interceden en el proceso fenomenológico, hacerlo visible es necesario para adentrarnos en los esenciales sin categorizar la experiencia vivida desde la teoría. Asimismo, implica una concienciación de los prejuicios y estereotipos del equipo con respecto al fenómeno de estudio.

Palabras clave

Participación política, Experiencia, Fenomenología, Hermenéutica.

Referencia recomendada

Folgueiras-Bertomeu, P., de-Ormaechea-Otalora, V., Salvà-Aige, A., y Comet-Donoso, M. (2022). La actitud fenomenológica de la epojé en un estudio sobre participación política de mujeres jóvenes. REIRE Revista d'Innovació i Recerca en Educació, 15(1), 1-10.

https://doi.org/10.1344/reire.35693

(C) 2021 Las autoras. Este artículo es de acceso abierto sujeto a la licencia Reconocimiento 4.0 Internacional de Creative Commons, la cual permite utilizar, distribuir y reproducir por cualquier medio sin restricciones siempre que se cite adecuadamente la obra original. Para ver una copia de esta licencia, visite https://creativecommons.org/licenses/by/4.0/CC) ( 


\title{
Títol (català)
}

L’actitud fenomenològica de l'epokhé en un estudi sobre participació política de dones joves

\section{Resum}

INTRODUCCIÓ. L'enfocament fenomenològic comporta una exploració constant. Per això resulta imprescindible que l'equip de recerca revisi els preconceptes que s'activen en relació amb el tema d'estudi. L'epokhé té un paper fonamental per comprendre la participació política de dones joves. El procés de revisió constant de les idees i els prejudicis és la part central en la recerca fenomenològica.

MÈTODE. S'exposen les quatre fases del procés de recerca, i amb més deteniment la primera part de la segona fase; en concret, en l'experiència de participació política de l'equip de recerca. Les reflexions sobre les experiències de les integrants de l'equip es fan a partir de relats, preguntes obertes i reunions sobre participació política.

RESULTATS i DISCUSSIÓ. Les idees i els prejudicis de l'equip de recerca intercedeixen en el procés fenomenològic. Fer-ho visible és necessari per endinsar-nos en l'essencial sense categoritzar l'experiència viscuda des de la teoria. Així mateix, implica una conscienciació dels prejudicis i estereotips de l'equip respecte al fenomen d'estudi.

\section{Paraules clau}

Participació política, Experiència, Fenomenologia, Hermenèutica.

\section{Title (English)}

The phenomenological attitude of the epoché in a study on the political participation of young women

\begin{abstract}
INTRODUCTION. The phenomenological approach involves constant exploration. To this end, it is essential for the research team to review the pre-concepts that are at work in relation to the object of study. The epoché has a fundamental role in understanding the political participation of women and young people. The process of the constant revision of ideas and prejudices is the central part of phenomenological research.
\end{abstract}

METHOD. The four phases of the research process are presented. The focus is then placed on the first part of the second phase, specifically on the research team's experience of political participation. The reflections on the experiences of the team members are based on stories, open questions and meetings on the issue of political participatio.

RESULTS and DISCUSSION. The ideas and prejudices of the research team intervene in the phenomenological process; making them visible is necessary in order to gointo the essentials without categorizing the lived experience from the theoretical point of view. It also requires an awareness of the team's prejudices and stereotypes regarding the phenomenon under study.

\section{Keywords}

Political participation, Experience, Phenomenology, Hermeneutics. 


\section{Introducción}

En este artículo se exponen las reflexiones generadas en torno a las experiencias y conocimientos en participación política de un equipo de investigación de la Universidad de Barcelona ${ }^{1}$ que está realizando un estudio sobre esta temática. La primera parte de esta investigación tiene como objetivo "Dar cuenta de las estructuras de significado de las experiencias vividas de participación política de mujeres de 18 a 35 años" ${ }^{2}$ y sigue una perspectiva metodológica cualitativa, en concreto, se trata de una investigación fenomenológica hermenéutica. Esta modalidad de investigación implica, entre otras cosas, una tarea previa al trabajo de campo con las informantes. En concreto, esta tarea forma parte de la segunda fase del estudio y consiste en la recogida y posterior reflexión de las experiencias en participación política por parte de las personas investigadoras. Según Van Manen (2003) "antes de solicitar a otros que nos brinden una descripción sobre un fenómeno a explorar, tendríamos que intentar hacer una primera nosotros, para poseer una percepción más puntual de lo que pretendemos obtener" (p. 82). El presente artículo se centra en este trabajo previo (primera parte de la segunda fase del estudio) y se ha llevado a cabo durante los meses de julio a diciembre de 2020.

\section{Justificación y fundamentación}

La fenomenología se basa en la comprensión de los fenómenos psicológicos y sociales y busca capturar las vivencias humanas, tal y como se experimentan - no como las conceptualizamos ni categorizamos - de una forma prereflexiva Pretende, así, llegar a las esencias ${ }^{3}$; es decir, a las estructuras de significado de la experiencia vivida. Se trata de "un proceso de apropiación, esclarecimiento y explicitación reflexiva de las estructuras de significado de la experiencia vivida" (Van Manen, 2003, p. 320).

Dentro de la perspectiva fenomenológica, en este trabajo nos centramos en la Fenomenología Hermenéutica $(\mathrm{FH})^{4}$ (Ayala-Carabajo, 2008, 2016; Ricoeur, citado en Domingo-Moratalla, 2001; Van Manen, 2003) que implica, también, la interpretación de la experiencia vivida mediante algún texto o mediante alguna forma simbólica. Para ello, la FH sigue una interacción dinámica entre seis actividades investigadoras (Van Manen, 2003):

1. Centrarse en un fenómeno que interese y que comprometa con el mundo a la persona que investiga.

2. Investigar la experiencia del modo en que se vive, y no tal como se conceptualiza.

3. Reflexionar sobre los aspectos esenciales que caracterizan el fenómeno.

4. Describir el fenómeno mediante el arte de escribir y reescribir.

5. Sostener una relación pedagógica firme con el fenómeno y orientada hacia él.

6. Equilibrar el contexto de la investigación siempre considerando las partes y el todo.

\footnotetext{
${ }^{1}$ El estudio está dentro de las líneas de investigación "participación” y “metodología” del Grupo de Investigación consolidado GREDI (https://www.ub.edu/gredi).

${ }^{2}$ La pregunta de investigación de esta primera parte del estudio es ¿Cuál es la experiencia vivida de participación política siendo mujer y joven?

${ }^{3}$ Buscar esencias, desde la perspectiva de la Fenomenología Hermenéutica, no guarda relación con el esencialismo categórico que ve las cosas en términos absolutos y a partir de sus propiedades físicas se derivan convicciones morales (Van Manen, 2003).

${ }^{4}$ La Fenomenología Descriptiva, por su parte, es pura descripción de la experiencia vivida. Fenomenología de Husserl y seguidores, por ejemplo, Giorgi.
} 
Una vez seleccionado el fenómeno de investigación -en nuestro caso, la participación política siendo mujer y joven - se debe investigar la experiencia tal y como se vive, para ello hay que diferenciar entre el conocimiento vivido prereflexivo y la percepción reflexiva (Ayala-Carabajo, 2017; Van Manen, 2003):

- El conocimiento vivido prereflexivo sobre el significado de algo (ejemplo: "vivencia del tiempo").

- La percepción reflexiva que tenemos de la estructura de significado de la experiencia vivida (ejemplo: “¿qué es el tiempo?”).

La FH busca hacerlas "accesibles al logos". Para ello, hace uso del conocimiento vivido prereflexivo y de la percepción reflexiva. Se podría decir que la FH trata de "superar" las falacias del realismo y del idealismo 5 .

Partiendo de esta distinción, en nuestro estudio recogemos el conocimiento vivido prereflexivo sobre "experiencias de participación política siendo mujer-joven" para, más tarde, llegar a una percepción reflexiva sobre las "estructuras de significado de las experiencias de participación política siendo mujer-joven".

En la investigación fenomenológica, el conocimiento vivido prereflexivo se recoge mediante los métodos empíricos descriptivos (relatos de experiencias, anécdotas, entrevista fenomenológica, etc.). Estos métodos tienen como objetivo principal recopilar descripciones de experiencias vividas (DEVs); en nuestro caso, DEVs de participación política siendo mujer-joven. Una vez recogidas estas descripciones, se da paso a la percepción reflexiva, lo cual implica un análisis de los aspectos estructurales o temáticos de la experiencia vivida; en nuestro caso, de los aspectos estructurales o temáticos de las experiencias de participación política siendo mujer-joven. Para ello, hacemos uso de los métodos reflexivos (análisis temático fenomenológico). El resultado, también, son descripciones fenomenológicas, pero, a diferencia de las anteriores, estas son ya resultado del análisis.

En todo este proceso, un aspecto clave es la actitud del investigador/a fenomenológico. La FH como enfoque de investigación aplicado (FHA), (Ayala-Carabajo, 2017; Kumar 2012; Van Manen, 2003; Wilson, 2012), requiere por parte de la persona investigadora una determinada orientación intelectual, afectiva, ética y filosófica, que le permita afrontar los obstáculos principales con los que se va a ir encontrando. Según Ayala-Carabajo (2017), estos obstáculos son:

a) Prejuicios

b) Excesiva teorización

c) Ejercicio de abstracción de las vivencias

Para afrontar estos obstáculos, la persona investigadora debe poner en práctica el método de la epojé (Lozano Díaz, 2006, Macías, 2018), término de origen griego adoptado por Husserl para referirse al acto de suspender tanto las creencias como la ciencia (Van Manen, 2016). Se trata de una actitud mental de poner entre paréntesis todo aquello que pueda impedir/obstaculizar la vía de acceso a las estructuras del significado del objeto de estudio. Poner entre paréntesis se utiliza, así, como una analogía con la matemática, lo que va entre paréntesis puede permanecer separado de las operaciones que se hacen por fuera de ellas (Van Manen, 2016).

La epojé - junto con la reducción en el sentido fenomenológico ${ }^{6}$ - representa grandes hallazgos en la fenomenología de Husserl. Tanto la epojé como la reducción tienen el sentido de retornar al mundo tal y como lo

\footnotetext{
5 “Es importante darse cuenta también de que la conciencia no puede describirse directamente (una descripción reduciría las ciencias humanas al estudio de la conciencia o de las ideas, es decir, la falacia del idealismo). De un modo similar, el mundo en sí mismo, sin referencia a una persona o conciencia experimentadora, no puede tampoco describirse directamente (un planteamiento así pasaría por alto que las cosas reales del mundo están siempre, de forma significativa, constituidas por seres humanos conscientes, es decir, la falacia del realismo)." (Van Manen, 2003, p. 28)

${ }^{6}$ El significado de "reducción fenomenológica" está orientado, irónicamente, contra el reduccionismo (codificar, categorizar, abstraer). La palabra reducción recupera en la fenomenología su sentido etimológico (re-ducere, retornar) (Van Manen, 2016).
} 
vivimos en la actitud prereflexiva. Así, todos los aspectos y los gestos metódicos de la epojé "sirven a este propósito: que uno se abra a la experiencia tal y como se vive -como ciertos fenómenos y acontecimientos se constituyen y se dan en la experiencia vivida-" (Van Manen, 2016, p. 252). Además, "haciendo epojé" podremos acercarnos más a "significados escondidos" y, sobre todo, tomar conciencia de nuestros propios juicios con relación a nuestra perspectiva del fenómeno a investigar (Macías, 2018).

\section{Objetivos y metodología}

Tal y como se ha comentado con anterioridad, en este artículo se presenta una reflexión sobre las experiencias y conocimientos teóricos de un equipo de investigación con respecto al fenómeno de estudio: la participación política. Esta reflexión responde a un trabajo previo que se realiza antes de iniciar el trabajo de campo con las participantes del estudio.

De acuerdo con la FHA, la finalidad última de esta tarea previa es tratar de poner entre paréntesis aquellos prejuicios, concepciones, ideas, etc. que el equipo de investigación tiene con respecto al fenómeno de estudio. Para ello, las investigadoras buscan:

- Identificar y reflexionar sobre concepciones, ideas, etc. que el equipo de investigación tiene sobre el fenómeno de estudio; participación política.

- Identificar y reflexionar sobre prejuicios que pueda tener el equipo de investigación en torno al objeto de estudio; participación política.

Este trabajo, previo al trabajo de campo, se corresponde con la primera parte de la segunda fase de una investigación fenomenológica que sigue la siguiente estructura:

\section{A. Primera fase}

Etapa previa o clarificación de presupuestos. En esta etapa se establecen las hipótesis, los preconceptos, los presupuestos desde donde parte el equipo de investigación que podrían intervenir en el estudio.

\section{B. Segunda fase}

\section{Primera parte}

Recoger la experiencia vivida. La experiencia de las personas investigadoras: en palabras de Max Van Manen (2003) "antes de solicitar a otros que nos brinden una descripción sobre un fenómeno a explorar, tendríamos que intentar hacer una primera nosotros, para poseer una percepción más puntual de lo que pretendemos obtener" (p. 82). El equipo de investigación realiza diferentes reuniones centradas en el concepto/las vivencias de participación política, escribe relatos $\operatorname{libres}^{7}$ sobre sus experiencias en participación política y responde a dos cuestiones sobre esta misma temática.

\section{Segunda parte}

La experiencia de las personas participantes en el estudio (mujeres de 18 a 35 años). Se recopilan descripciones sobre su experiencia vivida en participación política siendo mujer y joven. En esta fase se utiliza el relato descriptivo y la entrevista fenomenológica.

\footnotetext{
${ }^{7}$ En este momento de la investigación no seguimos los métodos empíricos y los métodos reflexivos de la FHA.
} 


\section{Tercera fase}

Reflexionar acerca de la experiencia vivida. En esta fase, el propósito está en intentar aprehender el significado esencial de algo. Para ello, se distingue entre: el tema fenomenológico (lo que vendría a ser la estructura de las experiencias), la descripción de la experiencia y la comprensión de la experiencia. Esto se realiza mediante los métodos reflexivos (análisis temático fenomenológico) ${ }^{8}$.

\section{Cuarta fase}

Escribir-reflexionar acerca de la experiencia vivida. La finalidad de esta fase es "integrar en una sola descripción todas las fisonomías individuales, con ello se determina la fisonomía grupal, es decir, la estructura que caracteriza al grupo estudiado" (Fuster Guillen, 2019, p. 228). Para Van Manen (2003), este proceso se denomina texto fenomenológico. Esta fase también implica "una revisión de fuentes fenomenológicas o confrontación del trabajo final con otros estudios del mismo enfoque" (Fuster Guillen, 2019, p. 228) (verificación). Esta etapa culmina con la elaboración del texto fenomenológico.

\subsection{Equipo de investigación}

El equipo de investigación está formado por seis investigadoras y una estudiante de doctorado de edades comprendidas entre los 29 y los 63 años. El equipo es diverso en cuanto a la relación que tienen con el objeto de estudio. En concreto:

- Tres de las investigadoras han investigado en participación política y tienen experiencia en el ejercicio de la participación política.

- Dos no han investigado en participación política y tienen poca experiencia en su ejercicio.

- Dos no han investigado en participación política, pero tienen experiencia en su ejercicio.

Desde el principio de la investigación, el equipo - de acuerdo con las fases del estudio- decide dedicar los primeros meses de la investigación a clarificar los conceptos, teorías, etc. que tiene cada una de las investigadoras con respecto al objeto de estudio para, más tarde, ponerlos entre paréntesis e identificar posibles prejuicios. Es decir, en este primer momento, el equipo de investigación empieza a aplicar el método de la epojé. Para ello, el equipo escribe relatos libres, responde a dos preguntas sobre participación política y realiza reuniones donde se intercambian opiniones, vivencias, teorías, etc. sobre participación política.

\section{Resultados y discusión}

\subsection{Sobre ideas de participación política}

Al retomar nuestros propios relatos, así como las respuestas dadas a las preguntas formuladas sobre participación política, y revisitar las grabaciones de los primeros encuentros del equipo, lo primero que se observa es una tendencia por parte de las investigadoras a ir hacia una excesiva teorización. A grandes rasgos, las personas del equipo que han investigado en esta temática justifican sus argumentaciones con estudios, teorías, etc. sobre participación política, mientras que las investigadoras que tienen menos experiencia empírica con el fenómeno de estudio recurren a la necesidad de rescatar conceptos y acordar una definición teórica consensuada. Al equipo

\footnotetext{
${ }^{8}$ Uno de los principales obstáculos en el momento de acercarnos al análisis temático fenomenológico es confundirlo con el análisis de contenido. Así, aunque ambos análisis son propios de los métodos cualitativos, estos son diferentes tanto en su filosofía como en su aplicación.
} 
le cuesta salir de los marcos teóricos y destinan muchos momentos de las reuniones a reforzar la necesidad de salirse de esos marcos, algo esencial en un estudio fenomenológico (Ayala-Carabajo, 2016; Van Manen, 2003).

Por otro lado, se observa que - en muchas ocasiones - las investigadoras abordan lo político como un adjetivo calificativo de la participación, o como dos constructos separados: "participación" y "política". Este aspecto es relevante si tenemos en cuenta que, en la investigación propuesta, la participación política se aborda como un tipo específico de experiencia vivida ${ }^{9}$. Por otro lado, destaca el hecho que varias de las personas del equipo ponen énfasis en el grado de conciencia. Es decir, para estas investigadoras, la participación política estaría unida al grado de concienciación que se tiene sobre la dimensión política de cualquier acción participativa. Este aspecto también es relevante si recordamos que la FHA busca, en un primer momento, ir al conocimiento prereflexivo; es decir, huir de la conciencia y del pensamiento reflexivo. En palabras de Merleau-Ponty (1962, p. 16-17): "El mundo no es lo que pienso sino lo que vivo".

También destacan algunas aportaciones que van en la línea de considerar que toda acción/participación es política, así como otras que reconocen que la justicia social es un aspecto inherente del propio concepto "participación política". Este aspecto nos conecta, durante las reuniones, de manera recurrente e insistente con la variable contextual que comporta para el equipo de investigación la participación política y, con ello, nos damos cuenta de la dificultad que como equipo tenemos para abstraernos del contexto. La investigación fenomenológica prescinde (o lo de un valor muy distinto) del contexto (Van Manen, 2003), aspecto complejo para la mayoría del equipo que profesionalmente destaca por tener una amplia trayectoria en investigación cualitativa, siendo lo contextual un elemento esencial/primordial en la mayoría de los métodos situados dentro de esta perspectiva metodológica (estudio de caso, etnografía, investigación narrativa, etc.).

También se observa un concepto vivido por una parte del equipo desde la implicación y el compromiso. El acto de participar políticamente está marcado por las trayectorias que las personas del equipo han vivido, desvelándose un componente biográfico en el propio acto participativo. De nuevo, como equipo volvemos a chocar con los principios de la fenomenología donde lo biográfico (Van Manen, 2003) no ocupa, en principio, un lugar relevante, ni significativo.

De la misma manera que nos ocurre con las variables contextuales y biográficas, nos pasa con la dimensión de género. El equipo de investigación comparte que la participación política se manifiesta y es modelada por esta dimensión. Sin entrar en este tema, puesto que sería objeto de otro artículo, este está siendo otro de los retos para las investigadoras, tratar de poner entre paréntesis teorías, aspectos, vivencias sobre género que como mujeres tienen. En este punto, queremos matizar que, como equipo, abrazamos también los avances en fenomenología crítica, perspectiva que asume que las llamadas "categorías de identidad" (raza, sexo, orientación sexual, etc.) hacen que las estructuras aparentemente universales como, por ejemplo, la percepción del espacio y el tiempo ${ }^{10}$, en la práctica no lo sean (Ahmed, 2007; Weiss et al., 2020).

\footnotetext{
${ }^{9}$ En concreto, el estudio está centrado en la experiencia vivida de participación política siendo mujer y joven.

${ }^{10}$ El tiempo y el espacio son, junto con otros, existenciales (temas Universales) dentro de la fenomenología. Los existenciales sirven para indagar en nuestro mundo de vida y, más concretamente, en los fenómenos particulares objeto de estudio (AyalaCarabajo, 2008; Van Manen, 2016). Por ejemplo, en nuestra investigación, nos debemos preguntar por: ¿Cómo pueden los existenciales de relación, cuerpo, espacio, tiempo, cosas y tecnología guiarnos al explorar las estructuras del sentido de la vivencia de la participación política siendo mujer y joven? En nuestro estudio, estas preguntas nos las hacemos desde la perspectiva de la fenomenología crítica.
} 
Por último, vinculado de manera más directa con la educación, las investigadoras comparten la visión democrática de la participación política, lo cual implica entenderla como un acto de creación dirigido a un proceso igualitario que actúa como proceso de llegada, no como punto de partida. Un proceso dinámico que le otorga un sentido procesual, susceptible ser educado/formado. De nuevo aquí, las investigadoras - acostumbradas a extraer de sus estudios propuestas - chocan con otra de las características de la fenomenología; en concreto, el hecho de tratarse de un método orientado hacia el cuestionamiento ${ }^{11}$, y no hacia la búsqueda de respuestas concretas, ni hacia el hallazgo de conclusiones, ni hacia el establecimiento de pautas, normas, generalizaciones. Orientarse hacia el cuestionamiento significa en una investigación fenomenológica "«cuestionar» algo de manera fenomenológica y, también, plantearse la pregunta de cómo es ese algo "realmente»" (Van Manen, 2003, p. 62). No obstante, a pesar de que la epistemología de la investigación fenomenológica topa con la elaboración de propuestas, en nuestro caso, educativas, el valor pedagógico y ético de la FHA debe estar presente a lo largo de todo el trabajo y es, ahí, donde la fenomenología nos ayuda a vislumbrar comprensiones e intuiciones educativas relacionadas con el fenómeno de estudio (Ayala-Carabajo, 2016). De hecho, de manera transversal, nuestro trabajo no pierde de vista en ningún momento el siguiente cuestionamiento: ¿Cuál es el sentido pedagógico/ético de la experiencia vivida de participación política siendo mujer-joven?

\subsection{Sobre prejuicios del equipo de investigación}

Vinculado con lo explicado en el apartado anterior, la posible rigidez con relación a aquello que entendemos como participación y, más en concreto, como participación política, responde a ciertas categorías previas. En este sentido, las conceptualizaciones y teorizaciones en relación con ella podrían dificultar otros modos de percibir las formas diversas y variables que toma la experiencia de participación y que la hacen, por tanto, difícilmente categorizable. De hecho, uno de los aspectos clave de la investigación fenomenológica, es también el tipo de análisis que se realiza, que es totalmente opuesto a emplear un conjunto de procedimientos interpretativos basados en técnicas cualitativas como; por ejemplo, el análisis de contenido, que procede creando y organizando la información en categorías deductivas e inductivas (Pérez Serrano, 1984).

Otra de las preocupaciones manifiestas tiene que ver con los liderazgos y la imagen del/la activista como aquel que debe conocer en profundidad lo que pasa y tener una idea formada sobre todo lo que acontece. Una suerte de activista/intelectual informado y conocedor que sabe siempre que decisiones hay que tomar y por donde hay que ir. Esta idea no se condice con la confianza en que cualquier persona puede participar en igualdad de condiciones más allá de su formación o capacidades.

Se expresa también en reiteradas ocasiones cierta incomodidad en relación con el método fenomenológico y, en particular, con el lugar que ocupa en ella la búsqueda de las esencias en la experiencia de participación política. Ciertas resonancias esencialistas se presentaron claramente como prejuicios sobre los que habría que trabajar y aclarar.

\footnotetext{
${ }^{11}$ Desde el "principio", cuando la investigadora/investigador fenomenológico inicia su estudio debe interrogarse en cuanto a la intencionalidad del estudio. Interrogarse sobre la intencionalidad implica no orientarse hacia el hallazgo de conclusiones o hacia el establecimiento de pautas, normas, generalizaciones. La intencionalidad "tender hacia" es un término filosófico que cobra especial protagonismo a partir de la irrupción de la fenomenología. No debe confundirse con intención (o voluntad).
} 


\section{Conclusiones}

Explorar las diferentes experiencias sobre participación política de las investigadoras del equipo a través del relato libre, nos hizo darnos cuenta de la dificultad de escribir sin tener consignas fenomenológicas concretas. Así, a partir de nuestra experiencia, acordamos la necesidad de no pedir a las informantes del estudio relatos libres sino pautados y bien dirigidos de acuerdo con nuestra pregunta de investigación.

Por otro lado, todo este proceso implicó el tomar conciencia del gran esfuerzo que teníamos que hacer para poner todo lo conversado, lo narrado, etc. entre paréntesis y nos ayudó a identificar muchos de los prejuicios que, sin saberlo, nos estaban condicionando en todo; inclusive, generando fuertes incomodidades con la aplicación del propio método. Salirnos de las teorías, entrevistar sin tener un mapa de categorías, etc. se nos antojaba -en la práctica- muy difícil, pero entrevistar de esa manera sabíamos que no era hacer FHA. Estamos en el camino, en el proceso de hacer fenomenología y, en ese "aprender haciendo", sí que hemos tomado decisiones importantes referentes a lo comentado a lo largo de este artículo.

La más relevante ha sido entender que en fenomenología no importa, a priori, que entienda cada una de las personas del equipo por participación política, tampoco importan todas las teorías, perspectivas, etc. escritas sobre la temática. Si tenemos en cuenta que la fenomenología implica volver a lo esencial, teníamos como equipo que volver a lo esencial en cuanto al concepto de participación política. Acorde con el método, esta decisión nos ha obligado a revisar la fenomenología política de Hannah Arendt, la fenomenología de la participación de Ashworth (1997), la fenomenología de la participación de Husserl (Estada-Mora, 2007) así como la propia etimología de ambas palabras. Estas revisiones nos han llevado a considerar en nuestro estudio la "participación política" como a aquella actividad/es del ciudadano/a cuando participa colectivamente en asuntos públicos que buscan el bien común. Esta decisión ha sido clave a la hora de seleccionar a las informantes del estudio.

También, trabajar con esta rigurosidad nos ha permitido poner en práctica desde el principio la objetividad y la subjetividad fenomenológica. La objetividad significa que la persona investigadora se mantiene fiel al objeto de estudio. Algo así como un guardián de la "auténtica" naturaleza del objeto. "Quiere mostrarlo, describirlo, interpretarlo y, a la vez, serle fiel, consciente de que la persona puede ser fácilmente engañada, confundida o fascinada por elementos ajenos" (Van Manen, 2003, p. 38). La subjetividad hace referencia a la capacidad de la persona investigadora de "penetrar" en el objeto de estudio y profundizar en toda su riqueza. Ayala-Carabajo también se refiere/entiende la subjetividad como la "firmeza" de la persona investigadora que trata de no dejarse influenciar/desviar por sus preconcepciones, prejuicios.

\section{Organismo colaborador}

Investigación financiada por el Ministerio de Ciencia e Innovación "Participación sociopolítica de jóvenes desde una perspectiva de género: aportes conceptuales, metodológicos y educativos" (PID2019-104804RB-I00).

\section{Referencias}

Ahmed, S. (2007). A phenomenology of whiteness. Feminist Theory, 8(2), 149-168. https://doi.org/10.1177/1464700107078139

Ashworth, P. D. (1997). The meaning of participation. Journal of phenomenological psychology, 28(1), 82-103. https://doi.org/10.1163/156916297X00040 
Ayala-Carabajo, R. (2008). La metodología fenomenológico-hermenéutica de M. Van Manen en el campo de la investigación educativa. Posibilidades y primeras experiencias. Revista de investigación educativa, 26(2), 409-430. https://revistas.um.es/rie/article/view/94001

Ayala-Carabajo, R. (2016). Formación de investigadores de las ciencias sociales y humanas en el enfoque fenomenológico hermenéutico (de van Manen) en el contexto hispanoamericano. Educación XX1, 19(2), 359-381. https://doi.org/10.5944/educXX1.16471

Ayala-Carabajo, R. (2017). Retorno a lo esencial: fenomenología hermenéutica aplicada desde el enfoque de Max van Manen. Caligrama.

Domingo-Moratalla, T. (2001). La fenomenología hermenéutica de Paul Ricoeur: mundo de la vida e imaginación. Investigaciones fenomenológicas: Anuario de la Sociedad Española de Fenomenología, 3, 291-302. https://doi.org/10.5944/rif.3.2001.5432

Estada-Mora, O. (2007). Fenomenología de la participación según E. Husserl. Estudios, 20, 296-304.

Fuster Guillen, D. E. (2019). Investigación cualitativa: Método fenomenológico hermenéutico. Propósitos y representaciones, 7(1), 201-229. http://dx.doi.org/10.20511/pyr2019.v7n1.267

Kumar, K. (2012). Interdisciplinary Health Research (IDHR): An analysis of the lived experience from the theoretical perspective of identity [Tesis doctoral, University of Sidney]. http://hdl.handle.net/2123/8858

Lozano Díaz, V. (2006). Hermenéutica y fenomenología: Husserl, Heidegger y Gadamer. Edicep.

Macías, G. (2018). Metodología para la Investigación Cualitativa Fenomenológica y/o Hermenéutica. Revista Latinoamericana de Psicoterapia Existencial, 17, 17-23.

Merleau-Ponty, M. (1962). Phenomenology of perception. Humanities Press.

Pérez-Serrano, G. (1984). El análisis de contenido en la prensa. La imagen de la Universidad a Distancia. Editorial UNED.

Van Manen, M. (2003). Investigación educativa y experiencia vivida. Idea Books.

Van Manen, M. (2016). Fenomenología de la práctica. Métodos de donación de sentido en la investigación y la escritura fenomenológica. Editorial Universidad del Cauca.

Weiss, G., Murphy, A., y Salamon, G. (2020). 50 Concepts for a Critical Phenomenology. Northwestern University Press.

Wilson, T. (2012). What can phenomenology offer the consumer? Marketing research as philosophical, method conceptual. Qualitative Market. Research: An International Journal, 15(3), 230-241.

https://doi.org/10.1108/13522751211231969 